ESAIM: COCV 19 (2013) 385-403

DOI: $10.1051 / \mathrm{cocv} / 2012014$
ESAIM: Control, Optimisation and Calculus of Variations

www.esaim-cocv.org

\title{
CONVEX SHAPE OPTIMIZATION FOR THE LEAST BIHARMONIC STEKLOV EIGENVALUE
}

\author{
Pedro Richrdo Simão Antunes ${ }^{1,2}$ And Filippo Gazzola ${ }^{3}$
}

\begin{abstract}
The least Steklov eigenvalue $d_{1}$ for the biharmonic operator in bounded domains gives a bound for the positivity preserving property for the hinged plate problem, appears as a norm of a suitable trace operator, and gives the optimal constant to estimate the $L^{2}$-norm of harmonic functions. These applications suggest to address the problem of minimizing $d_{1}$ in suitable classes of domains. We survey the existing results and conjectures about this topic; in particular, the existence of a convex domain of fixed measure minimizing $d_{1}$ is known, although the optimal shape is still unknown. We perform several numerical experiments which strongly suggest that the optimal planar shape is the regular pentagon. We prove the existence of a domain minimizing $d_{1}$ also among convex domains having fixed perimeter and present some numerical results supporting the conjecture that, among planar domains, the disk is the minimizer.
\end{abstract}

Mathematics Subject Classification. 35P15, 35J40, 65N80.

Received November 10, 2011. Revised February 13, 2012.

Published online January 10, 2013.

\section{INTRODUCTION}

Consider a plate, the vertical projection of which is the planar region $\Omega \subset \mathbb{R}^{2}$. A simple model for its elastic energy is

$$
J(u)=\int_{\Omega}\left(\frac{1}{2}(\Delta u)^{2}+(1-\sigma)\left(u_{x y}^{2}-u_{x x} u_{y y}\right)-f u\right) \mathrm{d} x \mathrm{~d} y,
$$

where $f$ is the external vertical load, $u$ is the deflection of the plate in vertical direction, and $\sigma$ is the so-called Poisson ratio which, for any material, satisfies the physical constraint

$$
-1<\sigma<1
$$

Keywords and phrases. Biharmonic operator, least Steklov eigenvalue, shape optimization, numerical method of fundamental solutions.

1 Departamento de Matemática, Universidade Lusófona de Humanidades e Tecnologias, av. do Campo Grande 376, 1749-024 Lisboa, portugal. pant@cii.fc.ul.pt

2 Grupo de Física Matemática da Universidade de Lisboa, Complexo Interdisciplinar, av. Prof. Gama Pinto 2, 1649-003 Lisboa, Portugal.

3 Dipartimento di Matematica, Politecnico di Milano, Piazza Leonardo da Vinci 32, 20133 Milano, Italy.

filippo.gazzola@polimi.it 
see $[19,22]$. Notice that for $\sigma>-1$ the quadratic part of the functional $J$ is positive. Moreover, first order derivatives do not appear in (1.1), which shows that the plate is free to move horizontally.

The variational formulation in (1.1) may be found in [13,17]. For hinged plates the natural boundary conditions lead to minimize $J$ in the Sobolev space $H^{2} \cap H_{0}^{1}(\Omega)$ and to the weak Euler-Lagrange equation

$$
\int_{\Omega}\left(\Delta u \Delta v+(1-\sigma)\left(2 u_{x y} v_{x y}-u_{x x} v_{y y}-u_{y y} v_{x x}\right)-f v\right) \mathrm{d} x \mathrm{~d} y=0
$$

for all $v \in H^{2} \cap H_{0}^{1}(\Omega)$. A formal integration by parts (see [15], Sect. 1.1.2 for the details) yields the strong Euler-Lagrange equation

$$
\begin{cases}\Delta^{2} u=f & \text { in } \Omega \\ u=\Delta u-(1-\sigma) \kappa u_{\nu}=0 & \text { on } \partial \Omega,\end{cases}
$$

where $u_{\nu}$ is the normal derivative of $u$ and $\kappa$ is the curvature of the boundary, with the sign convention that $\kappa \geq 0$ for convex boundary parts and $\kappa \leq 0$ for concave boundary parts. The differential equation $\Delta^{2} u=f$ is called the Kirchhoff-Love model $[17,22]$ for the vertical deflection of a thin elastic plate, whereas the boundary conditions are named after Steklov due to their first appearance in [30].

More generally, let $a \in C^{0}(\partial \Omega), f \in L^{2}(\Omega)$, and consider the boundary value problem

$$
\begin{cases}\Delta^{2} u=f & \text { in } \Omega \\ u=\Delta u-a u_{\nu}=0 & \text { on } \partial \Omega .\end{cases}
$$

We say that $u$ is a weak solution to (1.4) if $u \in H^{2} \cap H_{0}^{1}(\Omega)$ and

$$
\int_{\Omega} \Delta u \Delta v \mathrm{~d} x-\int_{\partial \Omega} a u_{\nu} v_{\nu} \mathrm{d} \omega=\int_{\Omega} f v \mathrm{~d} x \quad \text { for all } \quad v \in H^{2} \cap H_{0}^{1}(\Omega) .
$$

Let us mention that standard elliptic regularity results are available, see [15].

Consider the set $\mathcal{H}:=\left[H^{2} \cap H_{0}^{1}\right] \backslash H_{0}^{2}(\Omega)$ and define

$$
d_{1}(\Omega):=\min _{u \in \mathcal{H}} \frac{\int_{\Omega}|\Delta u|^{2} \mathrm{~d} x}{\int_{\partial \Omega} u_{\nu}^{2} \mathrm{~d} \omega} .
$$

If $\Omega$ is either smooth ( $\partial \Omega \in C^{2}$ ) or convex, then the minimum in (1.5) is achieved and the number $d_{1}$ in $(1.5)$ represents the least Steklov eigenvalue, namely the smallest constant value of $d$ for which the following problem admits a nontrivial solution

$$
\begin{cases}\Delta^{2} u=0 & \text { in } \Omega \\ u=\Delta u-\mathrm{d} u_{\nu}=0 & \text { on } \partial \Omega .\end{cases}
$$

In fact, there exists a countable set of eigenvalues. We refer to [11] for a fairly complete study of the spectrum and to [21] for a corresponding Weyl-type asymptotic behaviour.

The first purpose of the present paper is to describe the role of the least Steklov eigenvalue in bounded domains. In functional analysis, we immediately see from (1.5) that $d_{1}^{-1 / 2}$ plays the role of the norm of the compact linear operator

$$
\left.H^{2} \cap H_{0}^{1}(\Omega) \rightarrow L^{2}(\partial \Omega) \quad u \mapsto u_{\nu}\right|_{\partial \Omega} .
$$

From a physical point of view, we shall see that $d_{1}$ is the sharp upper bound for the function $a$ for the validity of the positivity preserving property for the hinged plate problem (1.4). Finally, we shall see that, thanks to Fichera's duality principle [12], $d_{1}$ also gives the optimal constant to estimate the $L^{2}(\Omega)$-norm of harmonic functions in terms of the $L^{2}(\partial \Omega)$-norm of their trace on the boundary. 
These applications make the problem of minimizing $d_{1}$ in suitable classes of bounded domains particularly interesting. The second purpose of the present paper is to survey the existing results and conjectures about this topic. Bucur et al. [9] showed that the minimization of $d_{1}$ among general domains (both of fixed measure or perimeter) leads to an infimum which is 0 and, of course, is not achieved. More recently, Bucur and Gazzola [8] proved the existence of an optimal domain when the minimization of $d_{1}$ is set up in the class of convex domains of given measure. However, the optimal convex domain (which is certainly not the disk, see (3.2) below) is still unknown. Here, we prove that there exists also a convex domain of fixed perimeter minimizing $d_{1}$. As we shall see, the measure and perimeter constraints lead to fairly different optimal shapes.

The third and main purpose of the present paper is to perform several numerical experiments in order to formulate new conjectures about the shape of the optimal planar convex domain. Among regular polygons of given measure it appears that the regular pentagon is the minimizer. We also compare the least Steklov eigenvalue of the regular pentagon with the Steklov eigenvalue of general (not necessarily regular) polygons having up to 8 sides (triangles, quadrilaterals, polygons, hexagons, heptagons, octagons); even in this enlarged class (with fixed measure), the regular pentagon appears to have the least Steklov eigenvalue. Finally, we also consider some "rounded polygons" (the so-called Reuleaux polygons) and the regular pentagon still seems to be a good candidate to be the absolute minimizer. Hence, quite surprisingly, for domains having fixed measure our results strongly suggest that the optimal planar shape is the regular pentagon. This result seems to be connected with a similar result by Bass et al. [6] for a different positivity preserving property for the biharmonic operator, see Remark 5.1 below. Then we switch to domains having fixed perimeter and we show that several "deformation maps" (which are not monotonic for domains having fixed measure) become monotonic. Moreover, for domains having fixed perimeter our results suggest that the optimal planar shape is the disk. This is related to a long-standing conjecture by Kuttler [18] which is false without the convexity constraint, see Theorem 3.1 below.

This paper is organized as follows. In Section 2 we explain in detail the applications of the least Steklov eigenvalue. In Section 3 we survey the existing results on the optimization of the least Steklov eigenvalue $d_{1}$ and we prove the existence of an optimal domain when the minimization of $d_{1}$ is set up in the class of convex domains of given perimeter. In Section 4 we briefly describe the application of the Method of Fundamental Solutions to the numerical solution of the least Steklov eigenvalue problem. In Sections 5 and 6 we compute numerically the least eigenvalue $d_{1}$ for several planar convex domains; we consider the class of convex domains with given area as well as the class of convex domains with given perimeter.

\section{The Role of the Least Steklov eigenvalue}

Throughout this section, $\Omega$ is a bounded domain of $\mathbb{R}^{n}(n \geq 2)$. Although the physical dimension is $n=2$, from a mathematical point of view it is straightforward to extend (1.3)-(1.5), and (1.7) to higher dimensions. In particular, (1.7) shows that $d_{1}$ has a deep meaning in functional analysis, regardless of the space dimension. Moreover, also the estimate in Section 2.2 shows that it is of some interest to consider the general case $n \geq 3$.

\subsection{Positivity preserving}

We are interested in finding conditions on $\Omega$ and on $a$ such that the assumption $f \geq 0$ implies that the solution $u$ to (1.4) exists and is positive. This is the so-called positivity preserving property, namely the property which ensures that the deflection $u$ has the same sign as the the vertical load $f$.

For any continuous function $\phi$, the notation $\phi \supsetneqq 0$ means $\phi(x) \geq 0$ for all $x$ and $\phi \not \equiv 0$. If $\phi$ is not continuous the same is intended a.e.

Denote by $d_{\partial \Omega}=d_{\partial \Omega}(x)>0$ the distance function from $x \in \Omega$ to $\partial \Omega$, then the following result holds

Theorem $2.1([7,14])$. Assume that $\partial \Omega \in C^{2}$. Let $a \in C^{0}(\partial \Omega), f \in L^{2}(\Omega)$, and consider the problem (1.4). There exists $\delta_{c}:=\delta_{c}(\Omega) \in[-\infty, 0)$ such that:

1. if $a \geq d_{1}$ and if $0 \supsetneqq f \in L^{2}(\Omega)$, then (1.4) admits no positive solutions; 
2. if $a=d_{1}$, then (1.4) admits a positive eigenfunction $u_{1}>0$ in $\Omega$ for $f=0$; Moreover, $u_{1}$ is unique up to multiples.

3. if $a \supsetneqq d_{1}$, then for all $f \in L^{2}(\Omega)$ there exists a unique solution $u$ to (1.4). If also $a \geq 0$ and $f \supsetneqq 0$, then the solution $u$ is strictly superharmonic in $\bar{\Omega}$;

4. if $\delta_{c} \leq a \supsetneqq d_{1}$, then $0 \supsetneqq f \in L^{2}(\Omega)$ implies $u \supsetneqq 0$ in $\Omega$;

5. if $\delta_{c}<a \supsetneqq d_{1}$, then $0 \supsetneqq f \in L^{2}(\Omega)$ implies $u \geq c_{f} d_{\partial \Omega}$ in $\Omega$ for some $c_{f}>0$;

6. if $a<\delta_{c}$, then there are $0 \supsetneqq f \in L^{2}(\Omega)$ with $0 \not \leq u$;

7. if $\Omega=B$, the unit ball, then $\delta_{c}=-\infty$.

Going back to the hinged plate model discussed in the Introduction, Theorem 2.1 yields the positivity preserving property for the hinged plate in planar convex domains. Recall that the physical bounds for the Poisson ratio are given in (1.2).

Corollary 2.2 ([24]). Let $\Omega \subset \mathbb{R}^{2}$ be a bounded convex domain with $\partial \Omega \in C^{2}$ and assume (1.2). Then for all $f \in L^{2}(\Omega)$ there exists a unique $u \in H^{2} \cap H_{0}^{1}(\Omega)$ minimizer of the elastic energy functional $J$ defined in (1.1). The minimizer $u$ is the unique weak solution to (1.3). Moreover, $f \supsetneqq 0$ implies that there exists $c_{f}>0$ such that $u \geq c_{f} d_{\partial \Omega}$ and $u$ is strictly superharmonic in $\bar{\Omega}$.

\subsection{A priori estimates for harmonic functions}

Let $\Omega \subset \mathbb{R}^{n}$ be a bounded domain with Lipschitz boundary $\partial \Omega$. Let $g \in L^{2}(\partial \Omega)$ and consider the problem

$$
\begin{cases}\Delta v=0 & \text { in } \Omega \\ v=g & \text { on } \partial \Omega\end{cases}
$$

We are here interested in the optimal constant $\delta_{1}(\Omega)$ for the a priori estimate

$$
\delta_{1}(\Omega) \cdot\|v\|_{L^{2}(\Omega)}^{2} \leq\|g\|_{L^{2}(\partial \Omega)}^{2} .
$$

In order to characterize variationally $\delta_{1}$, we introduce the space

$$
\mathbf{H}:=\text { closure of }\left\{v \in C^{2}(\bar{\Omega}) ; \Delta v=0 \text { in } \Omega\right\} \text { w.r.t. the norm }\|\cdot\|_{L^{2}(\partial \Omega)} .
$$

Then we define

$$
\delta_{1}=\delta_{1}(\Omega):=\min _{h \in \mathbf{H} \backslash\{0\}} \frac{\int_{\partial \Omega} h^{2} \mathrm{~d} \omega}{\int_{\Omega} h^{2} \mathrm{~d} x} .
$$

The minimum is achieved. To see this, combine the continuous map (for weakly harmonic functions) $H^{-1 / 2}(\partial \Omega) \subset L^{2}(\Omega)$ (see Thm. 6.6 in Chap. 2 in [20]) with the compact embedding $L^{2}(\partial \Omega) \subset H^{-1 / 2}(\partial \Omega)$.

Fichera's original principle [12] was stated in smooth domains, namely $\partial \Omega \in C^{2}$. Here we need the result for convex domains:

Theorem $2.3([9,12])$. If $\Omega \subset \mathbb{R}^{n}$ is open bounded with Lipschitz boundary, then $\delta_{1}(\Omega)$ admits a minimizer $h \in \mathbf{H} \backslash\{0\}$. If we also assume that $\Omega$ is convex then this minimizer is positive, unique up to a constant multiplier and $\delta_{1}(\Omega)=d_{1}(\Omega)$.

This principle may be further extended to nonconvex nonsmooth domains $\Omega$, provided $\partial \Omega$ satisfies the uniform outer ball condition, see [9]. 


\section{Minimization of the least Steklov eigenvalue}

In 1968, Smith [28] conjectured that a Faber-Krahn-type inequality holds for the least Steklov eigenvalue: For any bounded domain $\Omega \subset \mathbb{R}^{2}$, one has

$$
d_{1}(\Omega) \geq d_{1}\left(\Omega^{*}\right),
$$

where $\Omega^{*}$ denotes a ball such that $\left|\Omega^{*}\right|=|\Omega|$. Smith [28] also gives a proof of (3.1). However, as noticed by Kuttler and Sigillito, his proof contains a gap, see the "Note added in proof" in [29] where Smith also writes:

Although the result is probably true, a correct proof has not yet been found.

From [7] we know that $d_{1}(B)=n$, where $B$ is the unit ball in $\mathbb{R}^{n}$. Hence, in particular, for planar domains $\Omega$ of measure $\pi$ (as the unit disk), (3.1) would mean that $d_{1}(\Omega) \geq 2$. A couple of years later, Kuttler [18] showed that for the square $\Omega_{4}=(0, \sqrt{\pi})^{2}$ one has $d_{1}\left(\Omega_{4}\right)<1.9889 \ldots$ This estimate was subsequently improved in [11] by

$$
d_{1}\left(\Omega_{4}\right)<1.96256 .
$$

Therefore, (3.1) is false. For this reason, Kuttler [18] suggests a different and weaker conjecture.

Let $\Omega \subset \mathbb{R}^{n}$ be a smooth bounded domain such that $|\partial \Omega|=|\partial B|$, where $|\cdot|$ represents the $(n-1)$-Hausdorff measure. Then, $n=d_{1}(B) \leq d_{1}(\Omega)$.

Kuttler gives numerical results on some rectangles which support this conjecture. However, the next result states that it is not true that the ball has the smallest $d_{1}$ among all domains having the same perimeter.

Theorem 3.1 ([9]). Let $D_{\varepsilon}=\left\{x \in \mathbb{R}^{2} ; \varepsilon<|x|<1\right\}$ and let $\Omega_{\varepsilon} \subset \mathbb{R}^{n}(n \geq 2)$ be such that

$$
\Omega_{\varepsilon}=D_{\varepsilon} \times(0,1)^{n-2} ;
$$

in particular, if $n=2$ we have $\Omega_{\varepsilon}=D_{\varepsilon}$. Then, $\lim _{\varepsilon \rightarrow 0^{+}} d_{1}\left(\Omega_{\varepsilon}\right)=0$.

Note that with a suitable scaling, for any bounded domain $\Omega$ and any $k>0$, one has

$$
d_{1}(k \Omega)=k^{-1} d_{1}(\Omega) .
$$

The homogeneity property (3.3) shows that $d_{1}(k \Omega) \rightarrow 0$ as $k \rightarrow \infty$. This suggests that $d_{1}(\Omega)$ becomes "smaller" when the domain $\Omega$ becomes "larger". However, in view of Theorem 3.1, we know that the map $\Omega \mapsto d_{1}(\Omega)$ is not monotone decreasing with respect to domain inclusion. This fact makes the minimization problem very delicate. For this reason, and since the lower bound

$$
d_{1}(\Omega) \geq n \min _{x \in \partial \Omega} \kappa(x)
$$

is available when $\partial \Omega \in C^{2}$ has positive mean curvature $\kappa$ (see [11,26]), Bucur and Gazzola [8] have addressed the problem of minimizing $d_{1}(\Omega)$ among convex domains $\Omega$ of fixed measure. In this class, they proved that the optimal shape does exist.

Theorem $3.2([8])$. Among all convex domains in $\mathbb{R}^{n}$ having the same measure as the unit ball B, there exists an optimal one, minimizing $d_{1}$.

As suggested in [8], Theorem 3.2 should be complemented with the description of the optimal convex shape. This appears quite challenging since, in view of (3.2), we know that the optimal planar convex domain is not a disk. In Section 5 we show numerical results which suggest that the optimal shape could be the regular pentagon.

With similar arguments, complemented with a continuity result from [10], we prove here the following 
Theorem 3.3. Among all convex domains in $\mathbb{R}^{n}$ having the same perimeter as the unit ball $B$, there exists an optimal one, minimizing $d_{1}$.

Proof. Consider a sequence $\left\{\Omega_{m}\right\} \subset \mathbb{R}^{n}$ of convex domains having the same perimeter as the unit ball $B \subset \mathbb{R}^{n}$ such that $d_{1}\left(\Omega_{m}\right) \rightarrow \inf d_{1}$, where the infimum of $d_{1}(\omega)$ is taken among all convex sets $\omega$ whose perimeter equals $|\partial B|$. By [8], Lemma 5.3, we know that the sequence $\left\{\Omega_{m}\right\}$ is bounded (up to a translation, it is contained in some fixed ball of sufficiently large radius $R>0$ ), since otherwise $d_{1}\left(\Omega_{m}\right) \rightarrow+\infty$. This fact, combined with Blaschke selection theorem [27], Theorem 1.8.6, shows that, up to a subsequence, $\left\{\Omega_{m}\right\}$ converges in the Hausdorff metric to a convex domain $\Omega$. Hence, by [8], Theorem 5.1, (continuity of the map $\Omega \mapsto d_{1}(\Omega)$ with respect to Hausdorff convergence of convex domains), we know that

$$
d_{1}(\Omega)=\lim _{m \rightarrow \infty} d_{1}\left(\Omega_{m}\right)=\inf _{|\partial \omega|=|\partial B|} d_{1}(\omega) .
$$

In view of [10], Lemma 4.4, we know that $|\partial \Omega|=\left|\partial \Omega_{m}\right|=|\partial B|$ so that $\Omega$ is an absolute minimizer in the class of domains having perimeter equal to $|\partial B|$.

Also Theorem 3.3 should be complemented with the description of the optimal shape. In Section 6 we show numerical results which suggest that the optimal shape could be the disk.

\section{BRIEF DESCRIPTION OF THE NUMERICAL METHOD}

In this section we describe the application of the Method of Fundamental Solutions (MFS) for the numerical solution of the Steklov eigenvalue problem. The MFS is a meshfree numerical method for which the approximations are made by particular solutions built using the fundamental solution associated to the partial differential equation of the problem. For the Steklov eigenvalue problem, we consider the fundamental solution of the biharmonic equation

$$
\Phi(r)=r^{2} \log (r), \quad \text { where } \quad r=|x| .
$$

The MFS approximation is given by a linear combination

$$
u(x) \approx \tilde{u}(x)=\sum_{j=1}^{N} \alpha_{j} \Phi\left(\left|x-y_{j}\right|\right)+\sum_{j=1}^{N} \beta_{j} \partial_{n_{y_{j}}} \Phi\left(\left|x-y_{j}\right|\right),
$$

where $y_{j}, j=1, \ldots, N$ are distinct source points placed on an admissible source set (see e.g. [1]). For instance, when $\Omega$ is bounded and simply connected, we can take the boundary $\partial \widehat{\Omega}$ of an enclosing open set $\widehat{\Omega} \supset \bar{\Omega}$. By $\partial_{n_{y_{j}}} \Phi\left(\left|x-y_{j}\right|\right)$ we denote the normal derivative of the function $\Phi\left(\left|x-y_{j}\right|\right)$ at the point $y_{j} \in \partial \widehat{\Omega}$. We remark that the fundamental solution $\Phi$ in (4.1) is an analytic function except at the origin where it has a singularity and thus, the MFS approximation (4.2) is analytic in $\Omega$ because the source points do not intersect $\bar{\Omega}$.

The coefficients $\alpha_{j}$ and $\beta_{j}$ will be calculated by fitting the boundary conditions. As in [2] or [3] we define $m$ collocation points $x_{1}, \ldots, x_{m}$ almost equally spaced on $\partial \Omega$ and the approximated normal vectors

$$
\tilde{n}_{i}=\left(x_{i}-x_{i-1}\right)^{\perp}+\left(x_{i+1}-x_{i}\right)^{\perp},
$$

where $\left(v_{1}, v_{2}\right)^{\perp}:=-\left(v_{2}, v_{1}\right)$ for all $\left(v_{1}, v_{2}\right) \in \mathbb{R}^{2}$. The source points are defined by the sum

$$
y_{i}=x_{i}+\beta \frac{\tilde{n}_{i}}{\left|\tilde{n}_{i}\right|},
$$

for a constant $\beta>0$ chosen such that the source points remain outside $\bar{\Omega}$. Thus, imposing the boundary conditions of the Steklov eigenvalue problem at the collocation points we obtain a $(2 m) \times(2 m)$ linear system

$$
\begin{cases}\tilde{u}\left(x_{i}\right)=0, & i=1, \ldots, m \\ \Delta \tilde{u}\left(x_{i}\right)-d \tilde{u}_{\nu}\left(x_{i}\right)=0, & i=1, \ldots, m .\end{cases}
$$




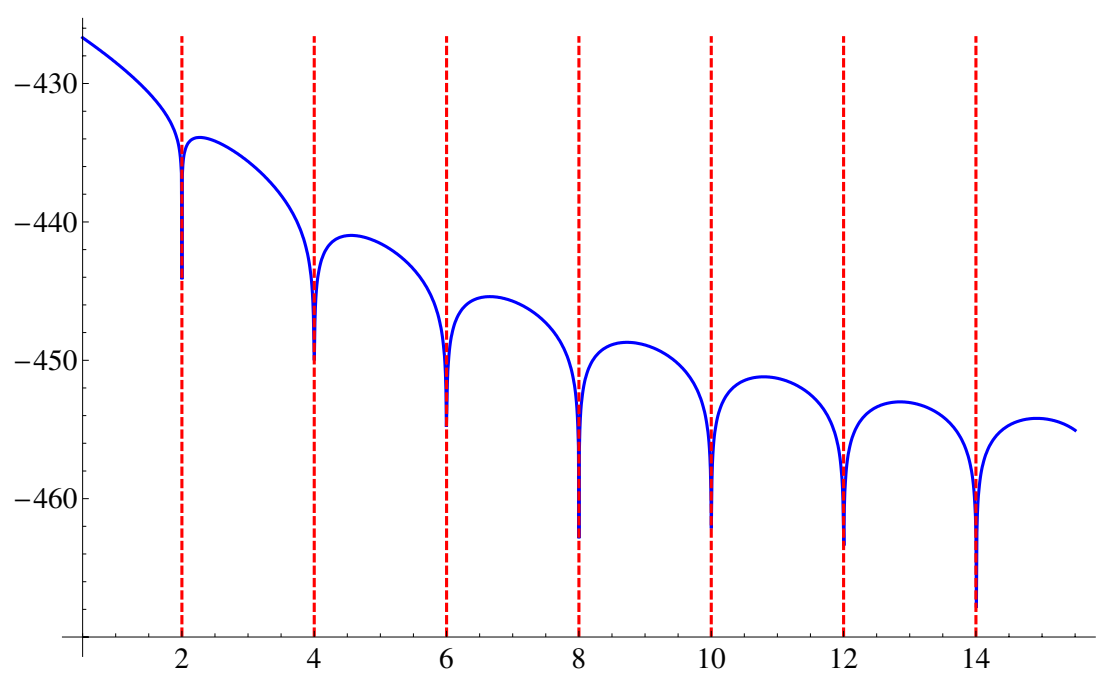

Figure 1. Plot of the graph of $g(d)$ for the unit disk.

We will denote by $\mathbf{M}(d)$ the matrix of this linear system. The numerical approximations for the Steklov eigenvalues are determined by seeking the values $d$ for which the matrix $\mathbf{M}(d)$ is not invertible, for example solving the nonlinear equation in $d, \operatorname{det}(\mathbf{M}(d))=0$. As in [2], we consider the real function

$$
g(d)=\log (|\operatorname{det}(\mathbf{M}(d))|)
$$

and search for the local minima of this function using an algorithm based on the golden ratio search method. Using the logarithmic function, the null determinant becomes a singularity that can be seen in the graph plot of the function $g$. To illustrate this fact, in Figure 1 we plot the function $g(d)$ obtained for the unit disk with $m=40$ and $\beta=0.4$. The dashed lines are the exact Steklov eigenvalues of the unit disk, see [11], Theorem 1.3. Once we have calculated an approximated Steklov eigenvalue $\widetilde{d}$, a corresponding eigenfunction is obtained using a collocation method with $m+1$ points, $x_{1}, \ldots, x_{m}$ on $\partial \Omega$, a point $x_{m+1} \in \Omega$ and an extra source point $y_{m+1} \in \bar{\Omega}^{C}$. The approximation is given by

$$
u(x) \approx \tilde{u}(x)=\sum_{j=1}^{m+1} \alpha_{m+1} \Phi\left(x-y_{m+1}\right)+\sum_{j=1}^{m} \beta_{m} \partial_{n_{y_{m}}} \Phi\left(x-y_{m}\right)
$$

and to exclude the trivial solution $\tilde{u}(x) \equiv 0$, the coefficients $\alpha_{j}$ and $\beta_{j}$ are obtained by solving the system

$$
\begin{cases}\tilde{u}\left(x_{i}\right)=0, & i=1, \ldots, m \\ \tilde{u}\left(x_{m+1}\right)=1 & \\ \Delta \tilde{u}\left(x_{i}\right)-\tilde{d} \tilde{u}_{\nu}\left(x_{i}\right)=0, & i=1, \ldots, m\end{cases}
$$

With the just described algorithm we computed the least Steklov eigenvalue $d_{1}(\Omega)$ for several planar convex domains. We performed a large scale study on convex polygons generated with a random procedure, as in a recent paper [5]. We considered the shape optimization problem both with area and perimeter constraints. In the next two sections we present the numerics that we gathered for both problems. 


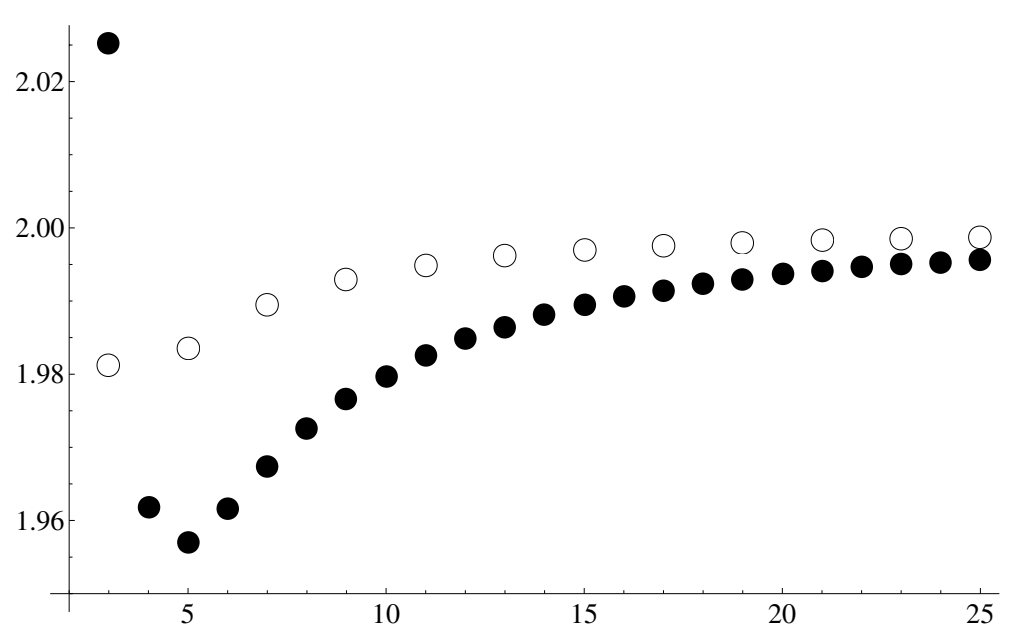

Figure 2. The maps $n \mapsto d_{1}\left(\Omega_{n}\right)$ (regular polygons $\bullet$ ) and $n \mapsto d_{1}\left(R_{n}\right)$ (Reuleaux polygons $\circ$ ).

\section{Results With area CONStraint}

\subsection{Regular polygons, reuleaux polygons}

Throughout this subsection we denote by $\Omega_{n}$ a regular $n$-polygon of measure $\pi$ having $n$ sides and by $D$ the unit disk. In the next table we display the numerically found values of $d_{1}\left(\Omega_{n}\right)$.

\begin{tabular}{|c|c|c|c|c|c|c|c|c|}
\hline$n$ & 3 & 4 & 5 & 6 & 7 & 8 & 9 & 10 \\
\hline$d_{1}\left(\Omega_{n}\right)$ & 2.02522 & 1.96179 & 1.95702 & 1.96164 & 1.96733 & 1.97255 & 1.97654 & 1.97974 \\
\hline
\end{tabular}

Several remarks appear naturally. First, it appears that the equilateral triangle $\Omega_{3}$ is the maximizer. Moreover, according to (3.2) we know that for the square $\Omega_{4}$ we have $d_{1}\left(\Omega_{4}\right)<1.96256$ while we find that $d_{1}\left(\Omega_{4}\right) \approx 1.96179$. Note also that the regular pentagon $\Omega_{5}$ appears as the minimizer, a first good reason to believe it is also the minimizer among general convex domains. Finally, it seems that $n \mapsto d_{1}\left(\Omega_{n}\right)$ tends monotonically to $2=d_{1}(D)$ for $n \geq 5$ and $n \rightarrow \infty$, see the dots $\bullet$ in Figure 2 .

Remark 5.1. Let $\Omega \subset \mathbb{R}^{2}$ be a bounded domain, let $c>0$ and let $f \in L^{2}(\Omega)$. Consider the equation $\Delta^{2} u+c u=$ $f$ in $\Omega$ under Navier boundary conditions $u=\Delta u=0$ on $\partial \Omega$. This problem represents a simply supported plate whose projection on the plane is $\Omega ; f$ is the external load, $c$ is the "stiffness of the resistance to deformation" whereas $u$ is the vertical deformation of the plate. One is again interested in the positivity preserving property, namely under which conditions on $c>0$ and $\Omega$ the assumption $f \geq 0$ implies that the solution $u$ exists and is positive. This problem was addressed by McKenna and Walter [23] and, some years later, intensively studied by Kawohl and Sweers [16]. It is known that there exists a maximal interval $\left(0, c^{*}(\Omega)\right]$ of values of $c>0$ for which the positivity preserving property holds. One is then interested in finding the optimal (largest) value of $c^{*}(\Omega)$ when $\Omega$ varies among convex planar domains of given measure. Numerical results obtained in [6], Table 1, show that, among regular polygons, the maximum is attained by the pentagon $\Omega_{5}$. Since $d_{1}(\Omega)$ is the threshold for $a$ in order to have the positivity preserving property in (1.4), see Section 2.1, one can wonder if these results are somehow connected.

If we add some constant curvature on the sides of $\Omega_{3}$ and we consider the so-called Reuleaux triangle $R_{3}$ [31], we numerically find $d_{1}\left(R_{3}\right) \approx 1.9812<d_{1}\left(\Omega_{3}\right)$. This suggests that one could lower the value of $d_{1}\left(\Omega_{5}\right)$ by taking the corresponding Reuleaux pentagon $R_{5}$ [31]. This does not seem to be the case. In Figure 2 we compare these values for polygons having an odd number of sides. It turns out that only for the triangle, the Reuleaux polygon decreases $d_{1}$. So, $\Omega_{5}$ remains a good candidate to be the absolute minimizer. 


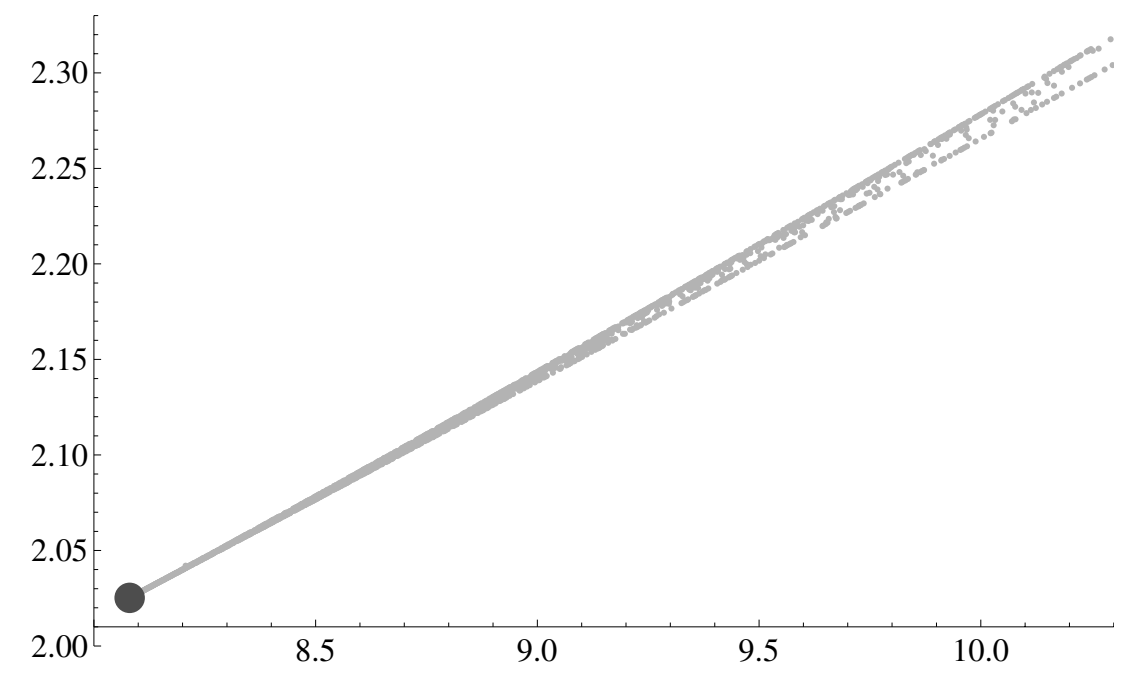

FiguRE 3. The correspondence $|\partial \Omega| \mapsto d_{1}(\Omega)$ for triangles $\Omega$ of measure $\pi$.

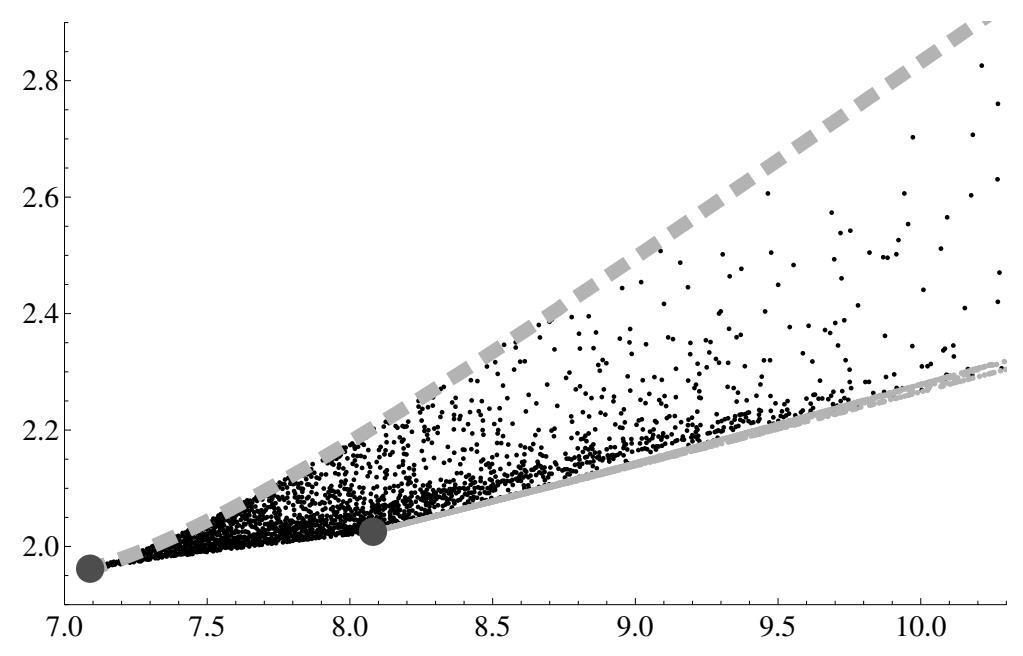

FiguRE 4 . The correspondence $|\partial \Omega| \mapsto d_{1}(\Omega)$ for triangles and quadrilaterals $\Omega$ of measure $\pi$.

\subsection{General polygons}

In Figure 3 we plot the results for the least Steklov eigenvalue $d_{1}$ of triangles of measure $\pi$, as a function of their perimeter. The minimum is attained by the equilateral triangle (large grey point), which has also the smallest perimeter. According to [5], Definition 2.1, we say that an isosceles triangle is subequilateral (resp. superequilateral) if the angle between its two equal sides is smaller (resp. larger) than $\pi / 3$. The cloud of points in Figure 3 is bounded above and below (respectively) by subequilateral and superequilateral triangles. Hence, all the triangles on the boundary of the cloud of points are isosceles triangles. The fact that the isosceles triangles appear as extremal sets in the class of triangles has already been observed in [4] when studying bounds for the spectral gap of the Laplacian.

In Figure 4 we add to the plot of Figure 3 similar results for quadrilaterals identified with black dots. The rectangles are marked with grey rectangles, and thus, for fixed perimeter (and area equal to $\pi$ ) they maximize 


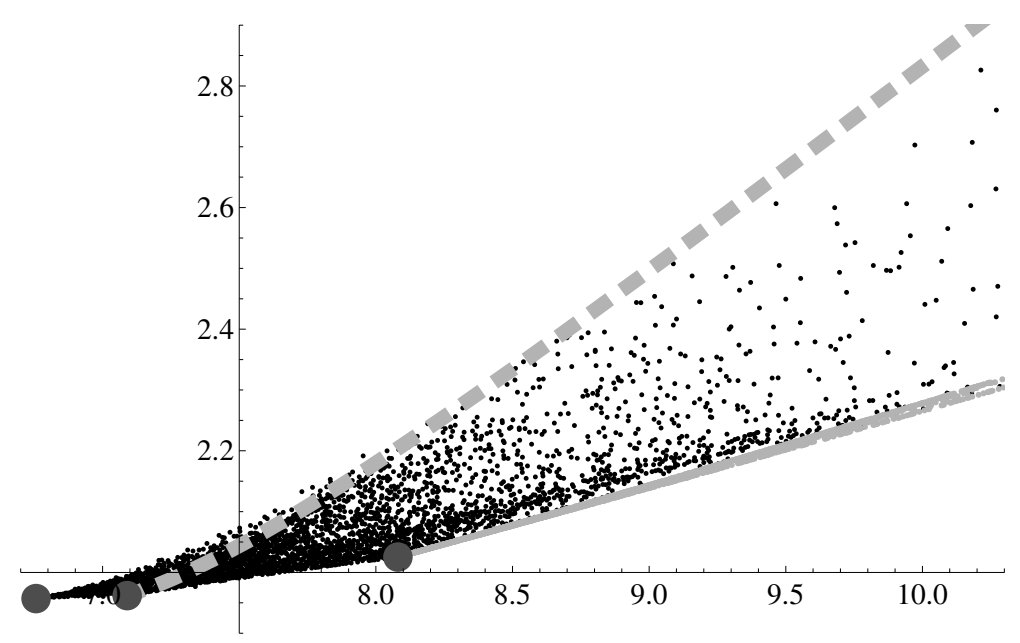

FiguRE 5 . The correspondence $|\partial \Omega| \mapsto d_{1}(\Omega)$ for polygons $\Omega$ of measure $\pi$ having $n \leq 5$ sides.

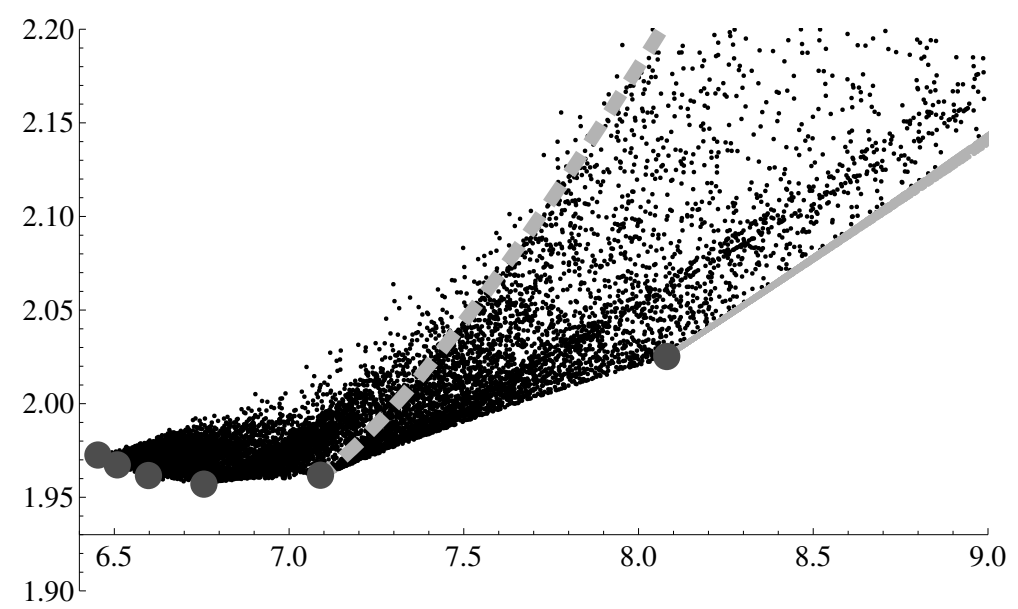

Figure 6 . The correspondence $|\partial \Omega| \mapsto d_{1}(\Omega)$ for $n$-polygons $\Omega$ up to $n=8$ (measure $\pi$ ).

the eigenvalue $d_{1}$ among polygons having at most 4 sides. On the other hand, for fixed perimeter and area equal to $\pi$, superequilateral triangles minimize $d_{1}$ among polygons having at most 4 sides. The square and the equilateral triangle are marked with a large grey point.

In Figure 5 we add the pentagons also identified with black dots. The regular pentagon is the absolute minimizer. Rectangles (grey rectangles) maximize the eigenvalue $d_{1}$ among polygons (of area equal to $\pi$ ) having at most 5 sides, only if the fixed perimeter is sufficiently large. Moreover, for fixed perimeter and area equal to $\pi$, superequilateral triangles minimize $d_{1}$ among polygons having at most 5 sides. Regular polygons are marked with a large grey point.

In Figure 6 we plot the same map for regular polygons with $n$ sides, for $3 \leq n \leq 8$; the black dots now represent hexagons, heptagons, and octagons. Our sample of polygons has 6100 triangles, 16000 quadrilaterals and $32500 n$-polygons with $5 \leq n \leq 8$. Again, we see that for fixed perimeter and area equal to $\pi$, superequilateral triangles minimize $d_{1}$ among polygons having at most 8 sides. Regular polygons are marked with a large grey point. 

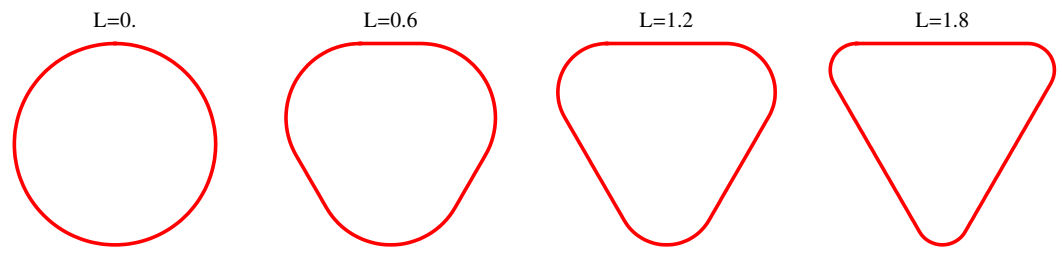

FIGURE 7. Deformation of the disk into a triangle.

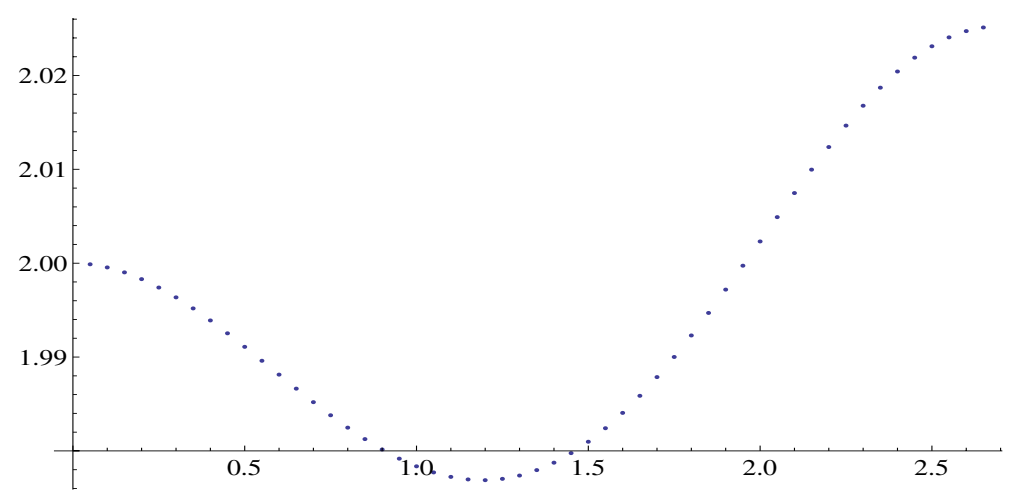

Figure 8 . The map $L \mapsto d_{1}(\Omega)$ when $\Omega$ is deformed as in Figure 7 .

We note that, even among these irregular polygons, the minimum eigenvalue is attained by the regular pentagon. We performed some optimization procedures of minimizing the least Steklov eigenvalue with convexity constraint and keeping area equal to $\pi$. In all experiments, we started with different octagons and our numerical method reached the regular pentagon. Thus, we believe that the regular pentagon is a good candidate to be the absolute minimizer among convex domains of given area.

\subsection{From the disk to regular polygons}

We also studied the evolution of the eigenvalue $d_{1}$ from the disk $D$ to the equilateral triangle $\Omega_{3}$. We start with three overlapped disks and then we move their centers on the vertices of an equilateral triangle having sides of length $L$. Consider the convex hull of the union of the three disks, so that we obtain convex domains such as in Figure 7.

Each domain is normalized in such a way that its measure is always equal to $\pi$. In Figure 8 we plot $d_{1}$ as a function of $L$ which varies between $L=0$ (disk $D$ ) and $L=2 \sqrt{\pi} / \sqrt[4]{3} \approx 2.69$ (equilateral triangle $\Omega_{3}$ ).

Similarly, if we start with four overlapped disks and then we move their centers on the vertices of a square having sides of length $L$, we consider the convex hull of the union of the four disks. Here $L$ varies between $L=0$ (disk $D$ ) and $L=\sqrt{\pi} \approx 1.77$ (square $\Omega_{4}$ ) and the corresponding map $L \mapsto d_{1}$ is represented in Figure 9 .

Note that both the maps in Figures 8 and 9 are not monotone.

We then deform in a similar way the unit disk $D$ into a regular pentagon $\Omega_{5}$ having same measure $\pi$. The geometric deformation is well described in Figure 10.

Here $L$ varies between $L=0(\operatorname{disk} D)$ and $L=\sqrt{4 \pi \sqrt{5-2 \sqrt{5}} / 5} \approx 1.35$ (pentagon $\Omega_{5}$ ) and the map $L \mapsto d_{1}$ is displayed in Figure 11.

Finally, in Figure 12 we plot the map $L \mapsto d_{1}$ from $L=0$ (disk) to $L=\sqrt{2 \pi / 3 \sqrt{3}} \approx 1.1$ (hexagon) for the same deformation.

Note that both the maps in Figures 11 and 12 are strictly decreasing. 


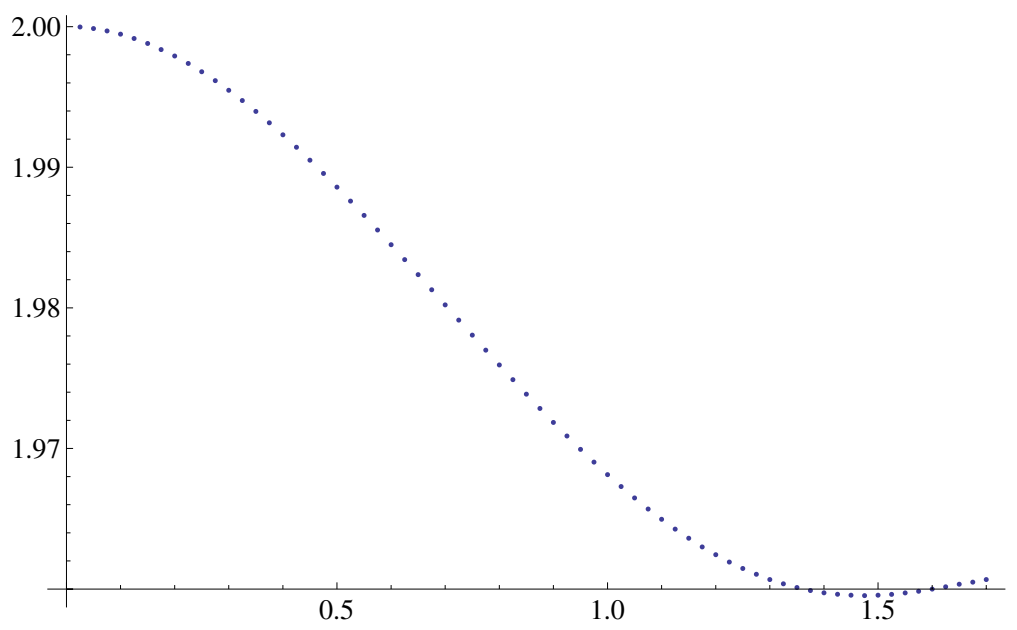

FiguRE 9 . The map $L \mapsto d_{1}$ when the disk is transformed into a square.
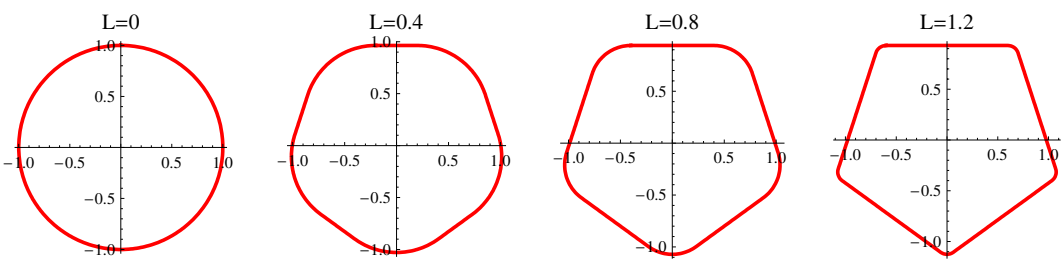

FiguRE 10. Deformation of the disk into a pentagon.

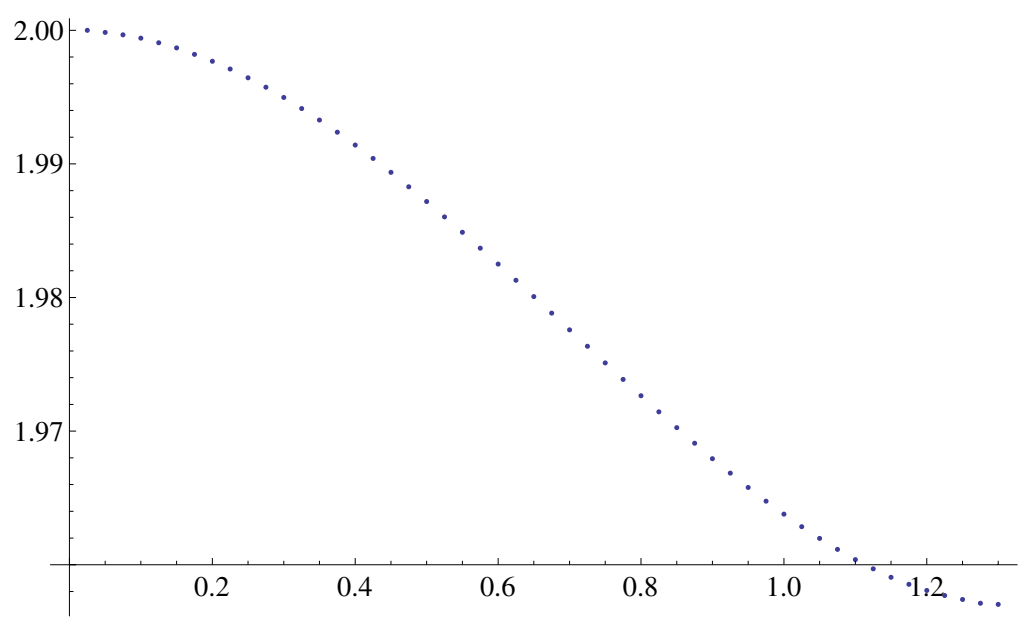

Figure 11. The map $L \mapsto d_{1}(\Omega)$ when $\Omega$ is deformed as in Figure 10 . 


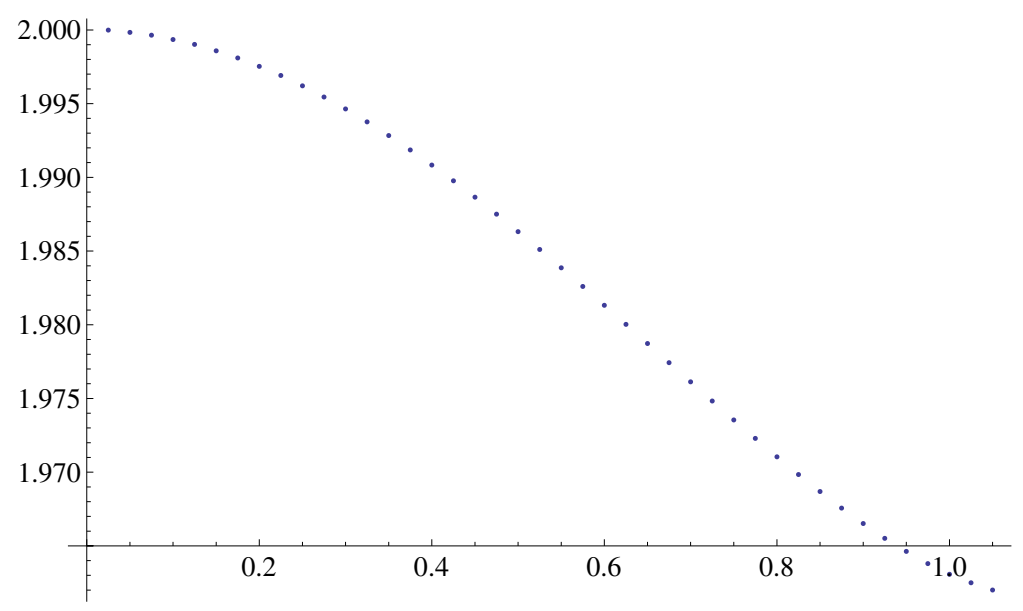

Figure 12. The map $L \mapsto d_{1}$ when the disk is transformed into an hexagon.

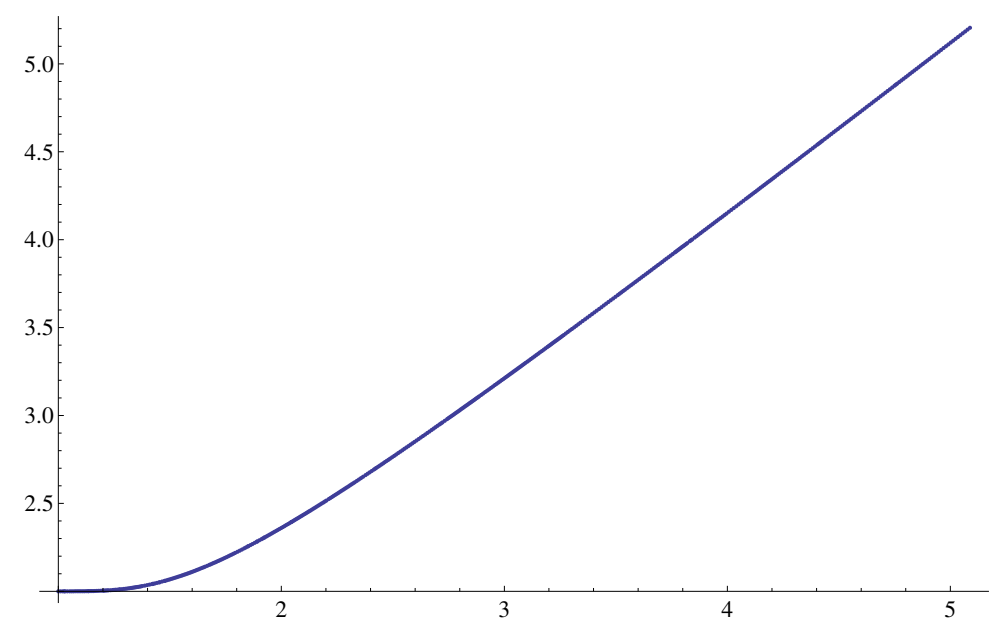

FiguRE 13. The map $R \mapsto d_{1}$ for ellipses from $R=1$ (disk) to $R \rightarrow \infty$ (segment).

\subsection{Ellipses and stadiums}

Now we consider ellipses with boundary defined by $\left\{\left(R \cos (t), \frac{1}{R} \sin (t)\right), t \in[0,2 \pi)\right\}$, for $R \geq 1$, which have area equal to $\pi$. In Figure 13 we plot the map $R \mapsto d_{1}$ which is strictly increasing. Note that by [25], formulaes (5.11)-(5.12), (see also [8], Lem. 5.3) $d_{1} \rightarrow+\infty$ as $R \rightarrow+\infty$.

Finally, we consider stadiums defined by the union of a rectangle whose sides have lengths equal to $L_{1}$ and $L_{2}$ and two semi-circles with diameter equal to $L_{2}$, as represented in Figure 14. Then, in order to keep area equal to $\pi$, we take $L_{1}=\frac{\pi}{L_{2}}-\pi \frac{L_{2}}{4}$. The disk corresponds to the case $L_{2}=2$, for which we obtain $L_{1}=0$. Again, in view of [25], we know that $d_{1} \rightarrow+\infty$ as $L_{2} \rightarrow 0$. In the left graph of Figure 15 we plot the map $L_{2} \mapsto d_{1}$ and in the right graph of the same Figure we present a zoom in a region near the disk. We can observe that the map is not monotone. 


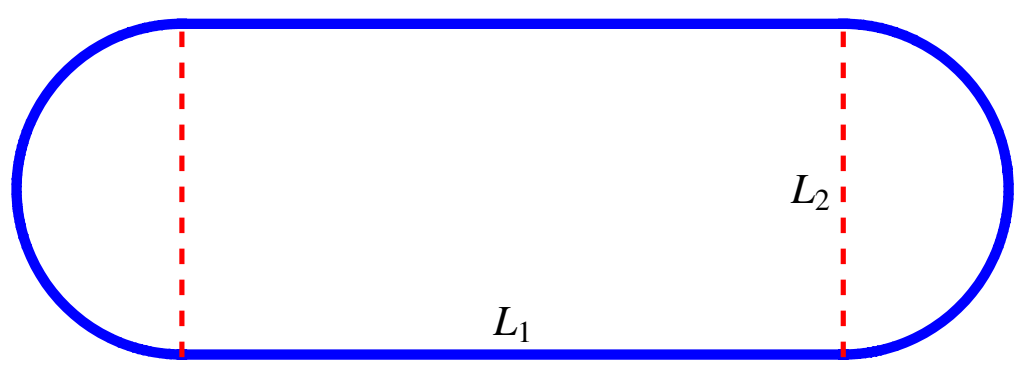

FiguRE 14. A stadium.
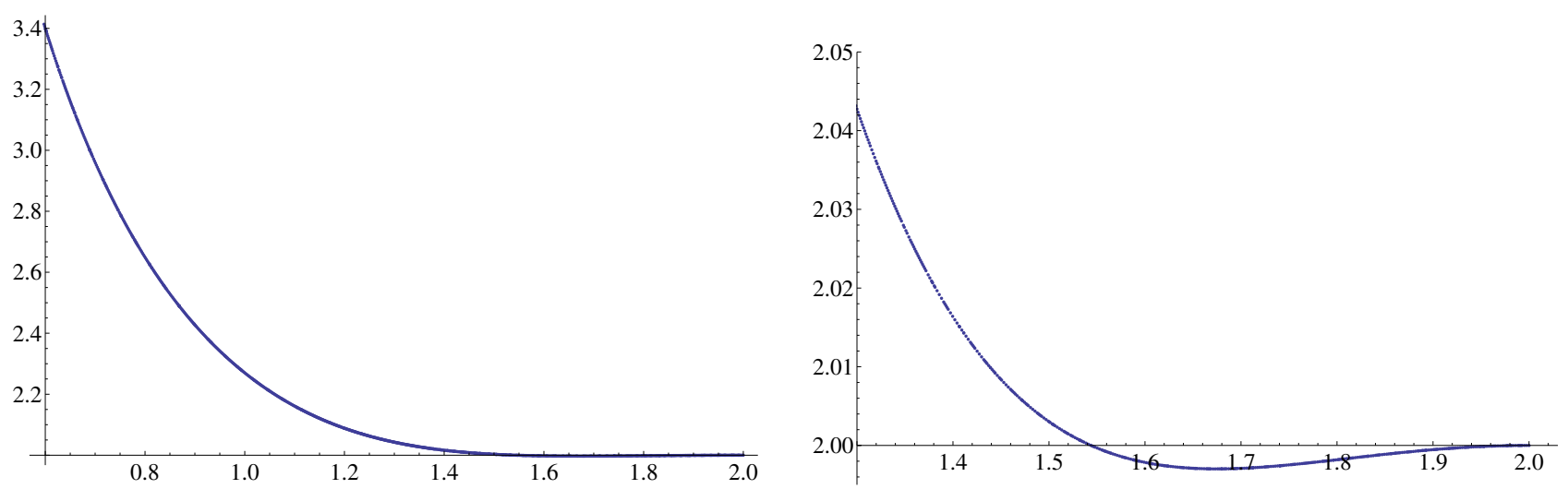

FigURE 15 . The map $L_{2} \mapsto d_{1}$ for stadiums of measure $\pi$.

\section{Results With PERIMeter CONSTRAint}

\subsection{Regular polygons}

In this subsection we present some numerical results for the minimization of the Steklov eigenvalue $d_{1}$ among convex planar domains with a given perimeter. All the domains considered in this section have perimeter equal to $2 \pi$. Note that for any $k>0$ we have

$$
\frac{|\partial(k \Omega)|^{2}}{|k \Omega|}=\frac{|\partial \Omega|^{2}}{|\Omega|} .
$$

This property, together with (3.3), enables us to transform the results for regular $n$-polygons $\Omega_{n}$ of measure $\pi$ into similar results for regular $n$-polygons $\Omega_{n}^{\sharp}$ of perimeter $2 \pi$ according to the formula

$$
d_{1}\left(\Omega_{n}^{\sharp}\right)=\sqrt{\frac{n}{\pi} \tan \frac{\pi}{n}} d_{1}\left(\Omega_{n}\right) .
$$

In the next table we show the numerical values of $d_{1}\left(\Omega_{n}^{\sharp}\right)$.

\begin{tabular}{|c|c|c|c|c|c|c|c|c|}
\hline$n$ & 3 & 4 & 5 & 6 & 7 & 8 & 9 & 10 \\
\hline$d_{1}\left(\Omega_{n}^{\sharp}\right)$ & 2.60458 & 2.21364 & 2.10443 & 2.05987 & 2.03791 & 2.02586 & 2.01830 & 2.01336 \\
\hline
\end{tabular}

In Figure 16 we plot the eigenvalue $d_{1}\left(\Omega_{n}^{\sharp}\right)$ for regular $n$-polygons with perimeter equal to $2 \pi$, as a function of the number of sides $n$ of the polygons for $3 \leq n \leq 25$. It may not be completely evident from the figure, but the sequence is monotone decreasing. 


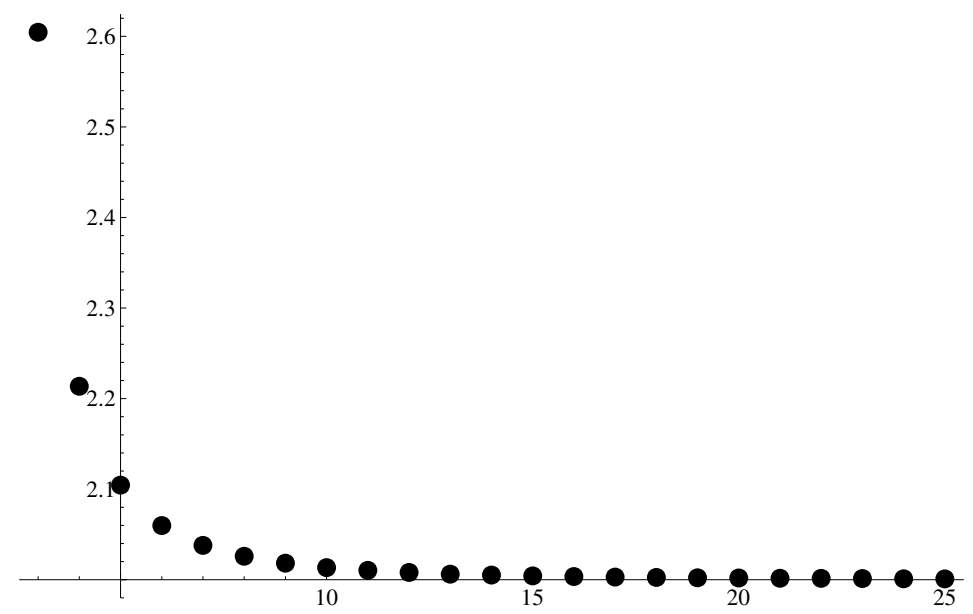

FIgURE 16 . The map $n \mapsto d_{1}\left(\Omega_{n}^{\sharp}\right)$ for regular $n$-polygons with perimeter equal to $2 \pi$.

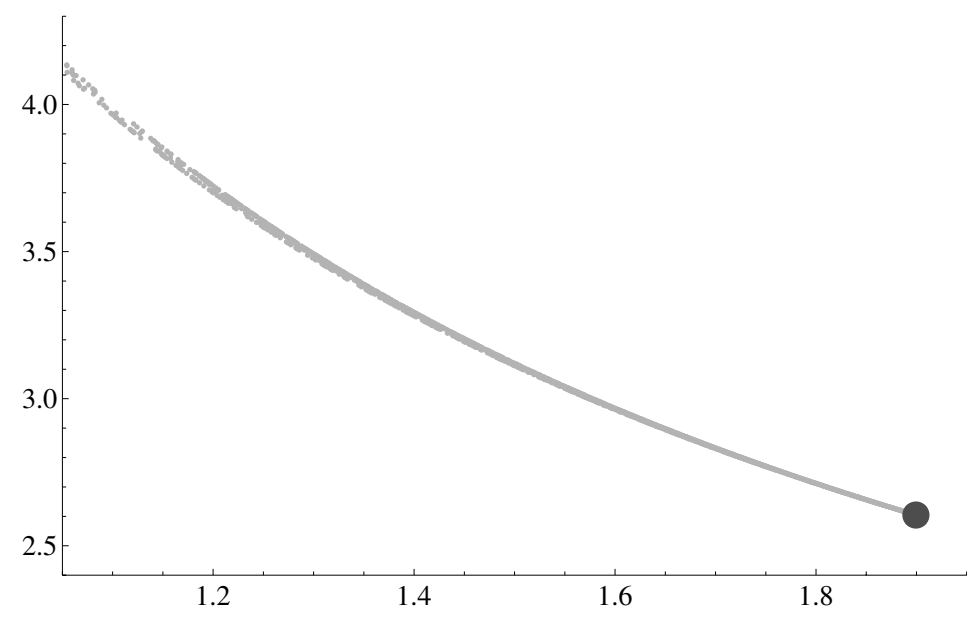

FiguRE 17. The correspondence $|\Omega| \mapsto d_{1}(\Omega)$ for triangles $\Omega$ with perimeter equal to $2 \pi$.

\subsection{General polygons}

In Figure 17 we plot results for the eigenvalue of triangles having perimeter equal to $2 \pi$, as a function of the area. The minimum is attained by the equilateral triangle (large grey point), which has the largest area. The region defined by the points is bounded above and below (respectively) by subequilateral and superequilateral triangles.

In Figure 18 we add the plot of similar results for quadrilaterals identified with black dots. The rectangles are again marked with grey rectangles, so that they maximize $d_{1}(\Omega)$ for polygons with at most 4 sides having perimeter $2 \pi$ and fixed area $|\Omega|$. Again, for fixed area and perimeter equal to $2 \pi$, superequilateral triangles minimize $d_{1}$ among polygons having at most 4 sides. The square and the equilateral triangle are marked with a large grey point.

In Figure 19 we add the same plot for pentagons also identified with black dots. The rectangles (grey rectangles) seem to maximize $d_{1}(\Omega)$ for polygons with at most 5 sides having perimeter $2 \pi$ only when the fixed area $|\Omega|$ is small. Note also that for fixed area and perimeter equal to $2 \pi$, superequilateral triangles minimize $d_{1}$ also among polygons having at most 5 sides. Regular polygons are marked with a large grey point. 


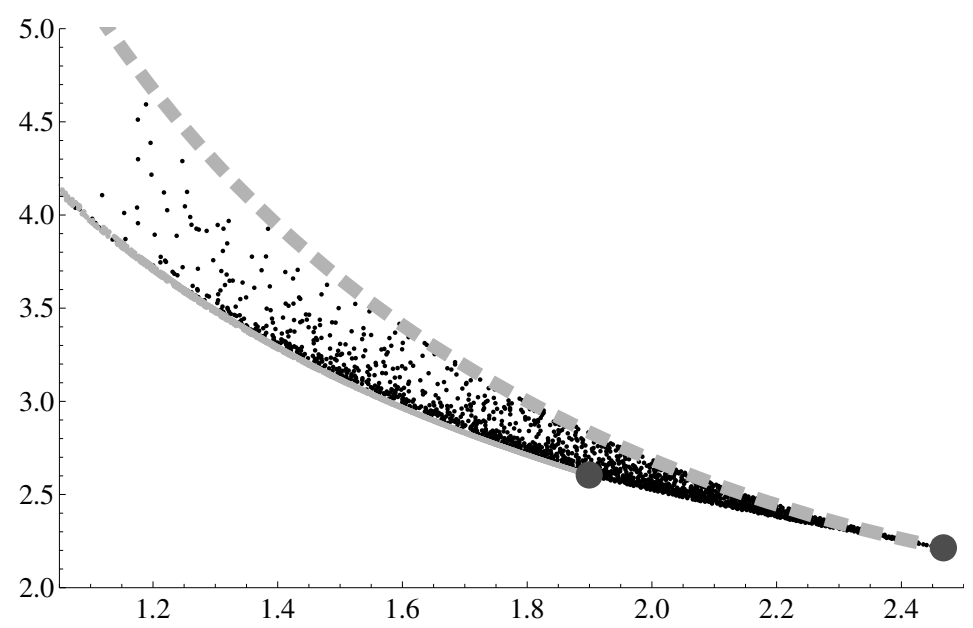

FiguRE 18. The correspondence $|\Omega| \mapsto d_{1}(\Omega)$ for quadrilaterals and triangles $\Omega$ with perimeter equal to $2 \pi$.

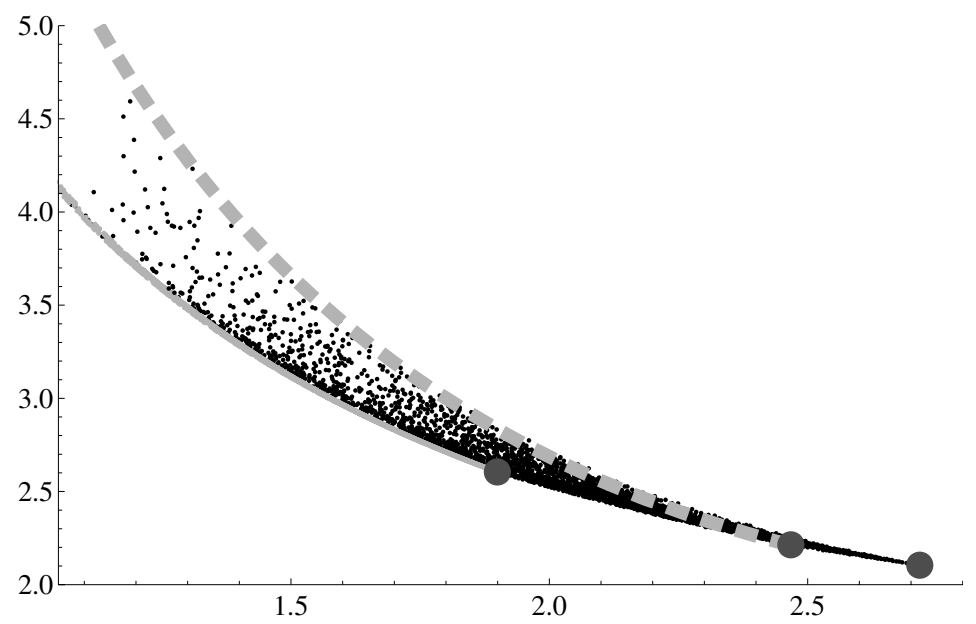

Figure 19. The correspondence $|\Omega| \mapsto d_{1}(\Omega)$ for polygons $\Omega$ with perimeter $2 \pi$ having $n \leq 5$ sides.

In Figure 20 we display similar results for regular polygons with $n$ sides, with $3 \leq n \leq 8$. The black dots represent hexagons, heptagons, and octagons, whereas the regular polygons are marked with large grey points. Also in this enlarged class rectangles (grey rectangles) seem to maximize $d_{1}(\Omega)$ only when the fixed area $|\Omega|$ is small whereas superequilateral triangles minimize $d_{1}(\Omega)$ for any area.

In all these classes of $n$-polygons we observe that the eigenvalue $d_{1}$ is minimized by the regular polygon $\Omega_{n}^{\sharp}$. We believe that the same type of property holds for other classes of $n$-polygons with $n>8$.

\subsection{From the disk to regular polygons}

Again we studied the evolution of the eigenvalue $d_{1}$ from the disk $D$ to regular polygons $\Omega_{n}^{\sharp}$, for $n=3,4,5,6$. We start with $n$ overlapped disks with radius equal to $R$ and move their centers on the vertices of a regular $n$-polygon having sides of length $L$. Then we consider the convex hull of the union of the $n$ disks and, for $0 \leq L \leq \frac{2 \pi}{n}$, we define $R=1-\frac{n L}{2 \pi}$ so that the domains have perimeter equal to $2 \pi$. In Figure 21 we display the maps $L \mapsto d_{1}$, for $n=3,4,5,6$. All these maps are monotone increasing. 


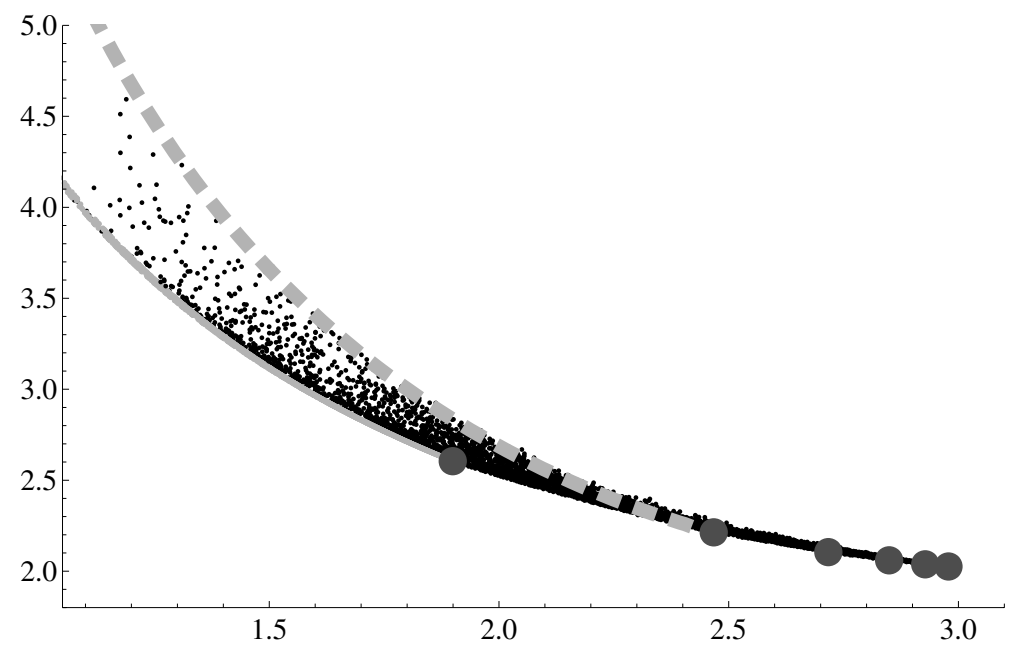

FiguRE 20. The correspondence $|\Omega| \mapsto d_{1}(\Omega)$ for $n$-polygons $\Omega$ up to $n=8$ (perimeter $2 \pi$ ).
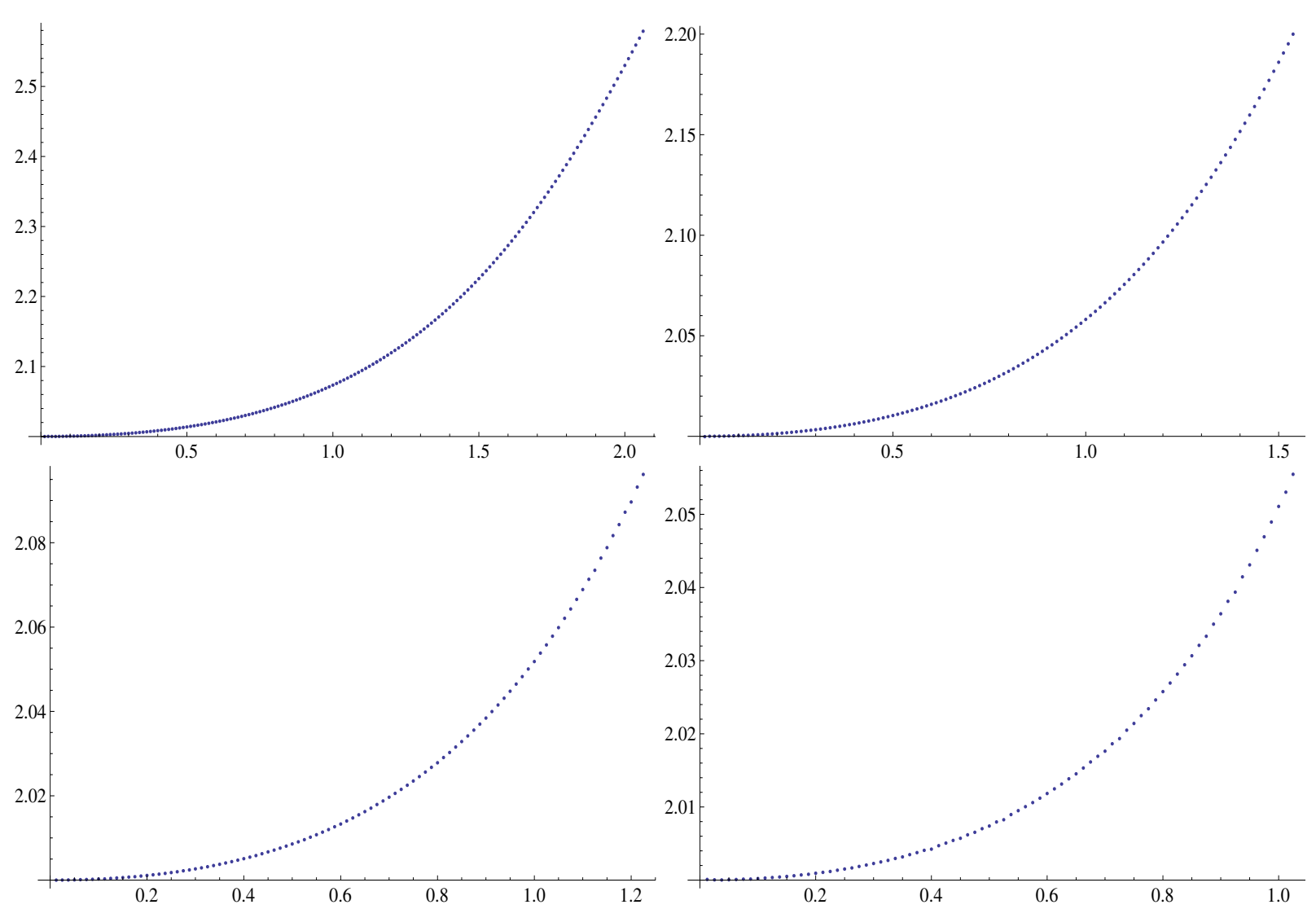

Figure 21. The maps $L \mapsto d_{1}(\Omega)$ when $\Omega$ is deformed from the disk to regular polygons $\Omega_{n}^{\sharp}$, $n=3,4,5,6$. 

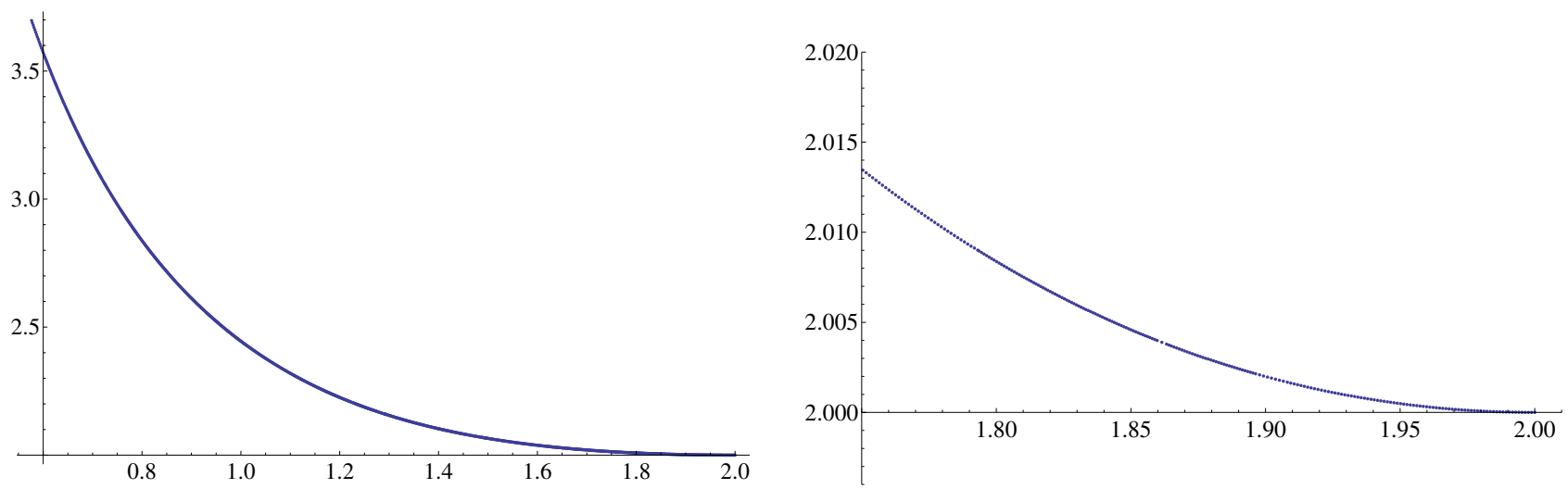

FiguRE 22. The map $L_{2} \mapsto d_{1}$ for stadiums with perimeter equal to $2 \pi$.

\subsection{Stadiums and conclusions}

Finally we considered the same map for stadiums as displayed in Figure 14. To keep perimeter equal to $2 \pi$ we define $L_{1}=\pi\left(1-\frac{L_{2}}{2}\right)$ and in Figure 22 we plot $d_{1}$ as a function of $L_{2}$ and a zoom of the same graph in a region near the disk. We can observe that the map is now monotone decreasing.

The monotonicity of the maps in Figures 16, 21 and 22 was not present in the case of domains of fixed measure, see Figures 2, 8, 9 and 15. This fact, together with all the numerical results we found, suggests that among all convex planar domains with a fixed perimeter, $d_{1}$ is minimized by the disk. Equivalently, for any convex planar domain $\Omega$ we would have

$$
d_{1}(\Omega) \geq \frac{4 \pi}{|\partial \Omega|}
$$

with equality if $\Omega$ is a disk.

Acknowledgements. The work of P.A. is partially supported by FCT, Portugal, through the scholarship SFRH/ $\mathrm{BPD} / 47595 / 2008$, and the scientific projects PTDC/MAT/101007/2008, PTDC/MAT/105475/2008 and PEstOE/MAT/UI0208/2011. The work of F.G. is partially supported by Progetto MIUR, PRIN 2008 "Aspetti geometrici delle equazioni alle derivate parziali e questioni connesse".

\section{REFERENCES}

[1] C.J.S. Alves, On the choice of source points in the method of fundamental solutions. Eng. Anal. Bound. Elem. 33 (2009) $1348-1361$.

[2] C.J.S. Alves and P.R.S. Antunes, The method of fundamental solutions applied to the calculation of eigenfrequencies and eigenmodes of 2D simply connected shapes. Comput. Mater. Cont. 2 (2005) 251-266.

[3] C.J.S. Alves and P.R.S. Antunes, The method of fundamental solutions applied to the calculation of eigensolutions for 2D plates. Int. J. Numer. Methods Eng. 77 (2008) 177-194.

[4] P. Antunes and P. Freitas, A numerical study of the spectral gap. J. Phys. A Math. Theor. 5 (2008) 055201.

[5] P.R.S. Antunes and A. Henrot, On the range of the first two Dirichlet and Neumann eigenvalues of the Laplacian. Proc. R. Soc. London Ser. A Math. Phys. Eng. Sci. 467 (2011) 1577-1603.

[6] R.F. Bass, J. Horák and P.J. McKenna, On the lift-off constant for elastically supported plates. Proc. Amer. Math. Soc. 132 (2004) 2951-2958.

[7] E. Berchio, F. Gazzola and E. Mitidieri, Positivity preserving property for a class of biharmonic elliptic problems. J. Differ. Equ. 320 (2006) 1-23.

[8] D. Bucur and F. Gazzola, The first biharmonic Steklov eigenvalue: positivity preserving and shape optimization. Milan J. Math. 79 (2011) 247-258.

[9] D. Bucur, A. Ferrero and F. Gazzola, On the first eigenvalue of a fourth order Steklov problem. Calc. Var. 35 (2009) $103-131$. 
[10] G. Buttazzo, V. Ferone and B. Kawohl, Minimum problems over sets of concave functions and related questions. Math. Nachr. 173 (1995) 71-89.

[11] A. Ferrero, F. Gazzola and T. Weth, On a fourth order Steklov eigenvalue problem. Analysis 25 (2005) 315-332.

[12] G. Fichera, Su un principio di dualità per talune formole di maggiorazione relative alle equazioni differenziali. Atti. Accut. Naz. Lincei 19 (1955) 411-418.

[13] K. Friedrichs, Die randwert und eigenwertprobleme aus der theorie der elastischen platten. Math. Ann. 98 (1927) 205-247.

[14] F. Gazzola and G. Sweers, On positivity for the biharmonic operator under Steklov boundary conditions. Arch. Ration. Mech. Anal. 188 (2008) 399-427.

[15] F. Gazzola, H.C. Grunau and G. Sweers, Polyharmonic boundary value problems. Lect. Notes Math. 1991 (2010).

[16] B. Kawohl and G. Sweers, On "anti"-eigenvalues for elliptic systems and a question of McKenna and Walter. Indiana Univ. Math. J. 51 (2002) 1023-1040.

[17] G.R. Kirchhoff, Über das gleichgewicht und die bewegung einer elastischen scheibe. J. Reine Angew. Math. 40 (1850) 51-88.

[18] J.R. Kuttler, Remarks on a Stekloff eigenvalue problem. SIAM J. Numer. Anal. 9 (1972) 1-5.

[19] R.S. Lakes, Foam structures with a negative Poisson's ratio. Science 235 (1987) 1038-1040.

[20] J.L. Lions and E. Magenes, Problèmes aux limites non homogènes et applications. Travaux et Recherches Mathématiques 3 (1970).

[21] G. Liu, The Weyl-type asymptotic formula for biharmonic Steklov eigenvalues on Riemannian manifolds. Adv. Math. 228 (2011) 2162-2217.

[22] A.E.H. Love, A treatise on the mathematical theory of elasticity, 4th edition. Cambridge Univ. Press (1927).

[23] P.J. McKenna and W. Walter, Nonlinear oscillations in a suspension bridge. Arch. Ration. Mech. Anal. 98 (1987) $167-177$.

[24] E. Parini and A. Stylianou, On the positivity preserving property of hinged plates. SIAM J. Math. Anal. 41 (2009) $2031-2037$.

[25] L.E. Payne, Bounds for the maximum stress in the Saint Venant torsion problem. Special issue presented to Professor Bibhutibhusan Sen on the occasion of his seventieth birthday, Part I. Indian J. Mech. Math. (1968/1969) 51-59.

[26] L.E. Payne, Some isoperimetric inequalities for harmonic functions. SIAM J. Math. Anal. 1 (1970) 354-359.

[27] R. Schneider, Convex bodies: the Brunn-Minkowski theory. Cambridge Univ. Press (1993).

[28] J. Smith, The coupled equation approach to the numerical solution of the biharmonic equation by finite differences I. SIAM J. Numer. Anal. 5 (1968) 323-339.

[29] J. Smith, The coupled equation approach to the numerical solution of the biharmonic equation by finite differences II. SIAM J. Numer. Anal. 7 (1970) 104-111.

[30] W. Stekloff, Sur les problèmes fondamentaux de la physique mathématique. Ann. Sci. Éc. Norm. Sup. 19 (1902) 191-259; $455-490$.

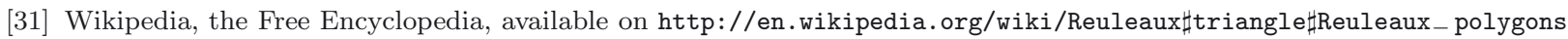

